\title{
Robust Control for Over-Actuated Vehicles*
}

\author{
Moad Kissai ${ }^{1}$, Bruno Monsuez ${ }^{1}$, Didier Martinez ${ }^{2}$, Xavier Mouton ${ }^{2}$, and Adriana Tapus ${ }^{1}$
}

\begin{abstract}
Many car manufacturers are equipping their vehicles with advanced systems to win the race towards autonomous vehicles. In order to ensure multiple objectives, different embedded systems influencing differently the same physical variable are often implemented in the same vehicle. Most of car manufacturers tend to activate one system at a time, especially when the low-level control of actuators is developed by different equipment suppliers, making the inner dynamics uncertain. However, this limits the potential of the vehicle. This paper discusses the feasibility of coordinating these systems when activated at the same time, while ensuring an acceptable robustness regarding the uncertain dynamics. Results showed good performance in severe maneuvers when combining robust control synthesis and optimization-based control allocation algorithms. Therefore, several embedded systems may be activated at the same time to expand the overall potential of the vehicle and deal with more difficult situations.

Index Terms-Robust Control, Chassis Systems, Vehicle Dynamics, Identification, Control Allocation, Online Optimization.
\end{abstract}

\section{INTRODUCTION}

The automotive sector is one of the most competitive ones. Different stakeholders are always rushing to propose original and advanced engineering solutions to improve the vehicle's safety and performance while ensuring the passengers' comfort. With the impressive progress of electronics and computer science, car manufacturers and equipment suppliers switched their focus to active systems since the 1970's [1]. These systems provide more flexibility in the design process, offer new features, can even avoid some accidents rather than mitigate the damages and so on. Due to their success, a natural motivation towards full autonomous vehicles was born and seduced the entire automotive sector. However, a full autonomous vehicle requires the ability to make its own decision like a human would do. Car manufacturers, taxi, and car-sharing services rely on Artificial Intelligence (AI) to overcome this complexity. The main philosophy is saying that most of accidents are caused by the lack of attention or fatigue of the driver, so we should replace this driver by an intelligence that does not loose attention and do not tire. The problem is that validating a complex autonomous system accurately and efficiently is non-trivial. Any visual perception system based on machine learning cannot be 100

\footnotetext{
*This work was supported by the Group Renault

${ }^{1}$ Moad Kissai, Bruno Monsuez, and Adriana Tapus are with ENSTA ParisTech, Department of Computer and System Engineering, Autonomous Systems and Robotics Team, 828 Boulevard des Marchaux, 91762 Palaiseau Cedex, France moad.kissai, bruno.monsuez, adriana.tapus\}ensta-paristech.fr

${ }^{2}$ Xavier Mouton, and Didier Martinez are with Group Renault, Chassis Systems Department, 1 Avenue du Golf, 78280 Guyancourt, France \{xavier.mouton, didier.d.martinez\}@renault.com
}

$\%$ accurate [2]. Hence, the system may fail for a specific input pattern and accurately estimating its failure rate can be extremely time-consuming [3]. One can affirm in this case that the AI will not be necessary better than humans, and could induce accidents that humans could have avoided.

A complementary solution that car manufacturers are working on is the over-actuation of the vehicles. Indeed, giving the vehicle new features as the ability of steering the rear wheels, distributing the brake torques or/and the engine torques differently among left and right tires and so one, can expand the vehicle's performance and generate new behaviors. For example, the Electronic Stability Program (ESP), which is based on the differential braking between right and left tires, has proven its effectiveness in dealing with severe emergency situations as obstacle avoidance [1]. The 4-Wheel Steering system can improve the vehicle's stability, or reduce the turning radius [4]. These systems cannot be controlled by the human driver directly. Therefore, an over-actuated vehicle that can control each one of its subsystems in harmony may exceed the human driver capabilities. Unfortunately, this imposes another complex problem, which is subsystems coordination. Not only that, the different subsystems come from different equipment suppliers and usually in a blackbox so the suppliers can protect their intellectual properties, which is known as the "openness" problem [5]. The car manufacturer has to implement different uncertain subsystems within the same vehicle, and provide a safer global vehicle motion control. The robustness of the overall control logic becomes a serious topic and should be ensured despite the different difficulties so the society can trust the future automated vehicles.

In this context, most of car manufacturers has opt for a "Downstream Coordination Approach" [5]. This means that each subsystem is kept within its own standalone controller, and then a coordination layer is added downstream these systems. In order to develop a coordination strategy in this configuration, interactions between the subsystems should studied before. Afterwards, automakers' engineers use their "expert knowledge" to develop coordination solutions. The work in [6] was based on this strategy and used Active Differential (AD), Electronic Stability Control (ESC), and Torque Vectoring (TV) to improve the vehicle lateral performances. A simple method based on prioritizing one system over another has been used. If the yaw torque demand can be satisfied by the AD, then the ESC and TV will not be activated. Otherwise, the rest of the yaw torque demand will be equally shared between the ESC and the TV systems. The systems are not activated at the same time, one system is prioritized according to the pre-studies. This raises the 
question of what is the goal of implementing several systems if only one system is activated at a time. In [7], a fuzzy logic scheme and weighting factors are used to coordinate the different systems. The controller agents computes combined control signals for the steering angle and the wheel torque depending on the targeted performances priority. The problem is not well settled and mathematically described. The rules are statistically/empirically developed and may never reach the optimum behavior. Moreover, all these methods relies on the pre-studies carried for a limited set of scenarios. As broad as the "expert knowledge" of automaker's engineers could be, we as humans cannot foresee all the possible scenarios.

The over-actuation problem has been encountered in the aeronautical sector before [8]. When conventional ganging methods were no longer able to handle advanced aircraft, researchers decided to formalize the problem mathematically, and develop optimization-based coordination approaches for flight control. Once the problem formalized, a high-level control can be designed to specify the motion of the center of gravity of the overall device. Then, the command can be optimally distributed to the subsystems. The coordination layer is then moved upstream the standalone controllers of subsystems. As you may expect, this approach was given the name of "Upstream Coordination Approach" in [5]. This method has been used in [9] to ensure fault-tolerance among chassis systems. The studied chassis systems are the Active Rear Steering (ARS) system and the brakingbased Vehicle Dynamics Control (VDC) system. Because of their high price, these systems are not redundant. Therefore, fault-tolerance methods using optimization-based control allocation algorithms were used to ensure a complementary between these systems, so if one system fails the other can take over. The method was validated via a co-simulation procedure using a high-fidelity vehicle model provided by LMS Imagine.Lab AMESim ${ }^{\circledR}$. In [10] and [11], energy consumption has been used as a criterion to be added to allocation precision and stability, proving that control allocation algorithms have the potential to fulfill multiple objectives. The authors in [12] have raised the question of ensuring qualitative objectives when allocating the commands. This is especially relevant for autonomous vehicles where comfortbased systems should be prioritized while monitoring the vehicle's safety. Authors have shown the ability to generate multiple sensations in an autonomous vehicle equipped by an Electric Power-Assisted Steering (EPAS) system, Torque Vectoring in front and rear, and an ESP. In this research also a co-simulation procedure using a high-fidelity vehicle model has been adopted. However, for new advanced embedded systems provided by the different equipment suppliers, their dynamics influenced by hidden control logic in black boxes can be uncertain, and even high-fidelity vehicle models can fail to depict the real behavior of the over-actuated vehicle.

The purpose of this paper is to develop a robust control logic for an over-actuated vehicle with uncertain embedded systems. Experiments were conducted on an over-actuated vehicle to isolate the influence of each subsystem apart. The control allocation algorithm is then adapted to fit the interfaces of each module. Results showed the limit of highfidelity software to depict the true dynamics of vehicles equipped with advanced systems. The controllers should be more sophisticated to fit the real dynamics of each actuator and optimize the overall behavior of the vehicle. The rest of the paper is then structured as follows: We start in Section II by presenting the modeling methods used for both robust control synthesis and control allocation design. In Section III, the robust control synthesis process is described. Control allocation algorithms are detailed in Section IV. Results are shown and discussed in Section V. Conclusions and future works are outlined in Section VI.

\section{MODELING METHODS}

Model Based Design (MBD) methodology has proven its effectiveness in control system development [13]. This makes a comprehensive design approach possible by developing models to represent the behavior of the plant to be controlled. However, the controller in this case is very related to the model. If the model is not accurate enough, the controller may fail to stabilize the plant. A proper way to proceed, is to first experimentally identify the dynamics involved, and then ensure the robustness of the control logic while keeping an acceptable level of performance.

However, for the control allocation algorithm, the commands have to be of the same unit, while the subsystems can be very different. In our case, the vehicle is equipped by both the 4-Wheel Steering (4WS) system and the braking-based Vehicle Dynamics Control (VDC) system. Note that the first system, which enables the steering of the rear wheels, can influence the yaw rate of the vehicle. The VDC system can generate different brake torques at the right wheels than at the left wheels. This generates different longitudinal tire forces at the corners of the vehicle which create a yaw moment. Consequently, the VDC can also influence the yaw rate of the vehicle. The influence of each chassis system should be first identified in order to manage their interactions.

\section{A. Experimental Identification}

In order to synthesize a robust high-level controller, the first stop is to identify the vehicle dynamics and its subsystems whenever it is possible. Normally, any subsystem will influence the tire forces then the vehicle dynamics. A high-level controller would be synthesized in the base of the vehicle dynamics, the control allocation algorithm should distribute optimally tire forces, and then a low-level control layer should control each subsystem apart. Unfortunately, we do not have access to tire forces signals online in passenger cars. Fig. 1 illustrates this problem. In Fig. 1, the accessible signals are in green, and the inaccessible one are in red, where:

- $\dot{\psi} \quad$ : yaw rate of the vehicle,

- $F_{x_{i, j}}$ : longitudinal force at the $i-j^{1}$ tire,

- $F_{y_{i, j}}$ : lateral force at the $i-j$ tire,

- $T_{b_{i, j_{r e q}}}$ : requested brake torque at the $i-j$ wheel,

${ }^{1} i=f$ for "front" or $r$ for "rear", and $j=l$ for "left" or $r$ for "right". 


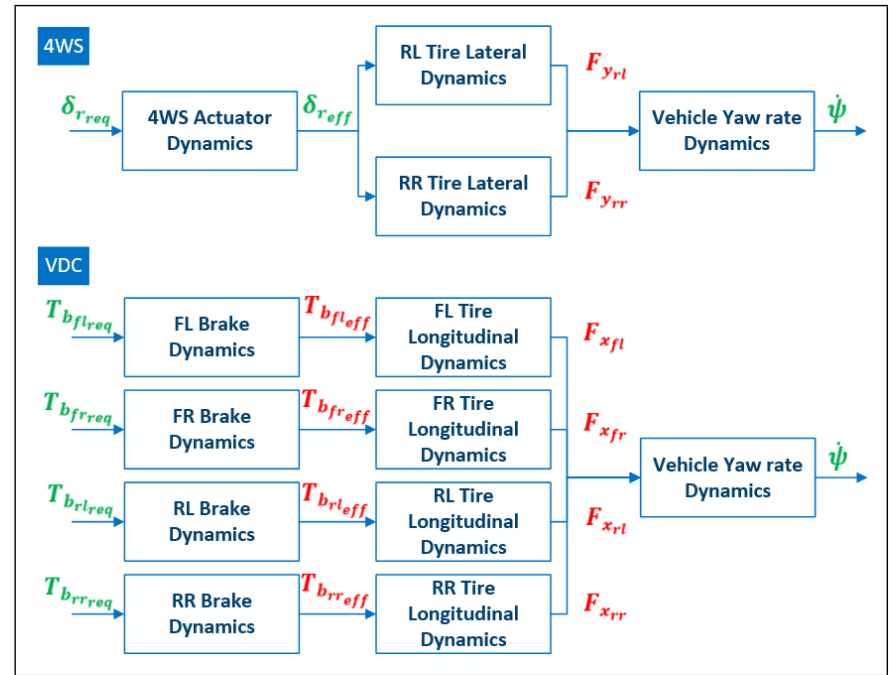

Fig. 1. The identification problem in an over-actuated vehicle.

- $T_{b_{i, j} \text { eff }}$ : effective brake torque at the $i-j$ wheel,

- $\delta_{r_{r e q}}$ : requested rear steering angle,

- $\delta_{r_{e f f}}$ : effective rear steering angle.

Note that also the effective brake torques cannot be measured. The good news is that we are able to measure both the input and output of the 4WS system. Therefore, by activating only the 4WS system we can isolate the vehicle dynamics from the actuator dynamics. Then, in the process of identifying the VDC dynamics, we can deduce the vehicle dynamics already identified, to isolate the subsystem dynamics.

1) The $4 \mathrm{WS}$ system identification: From previous research on vehicle dynamics [14],[15], we know that the vehicle dynamics depend on the speed. The experiments should be carried out using different speed values. Here, we apply a step to the 4WS system, and we measure both the effective rear steering angle $\delta_{r_{e f f}}$ and the yaw rate of the vehicle $\dot{\psi}$ to evaluate the influence of the 4WS actuator on the vehicle. We use afterwards the System Identification app of Matlab ${ }^{\circledR}$ to identify the dynamic models using input/output data. For a speed of $70 \mathrm{~km} / \mathrm{h}$ for example, we obtain the Fig. 2. We

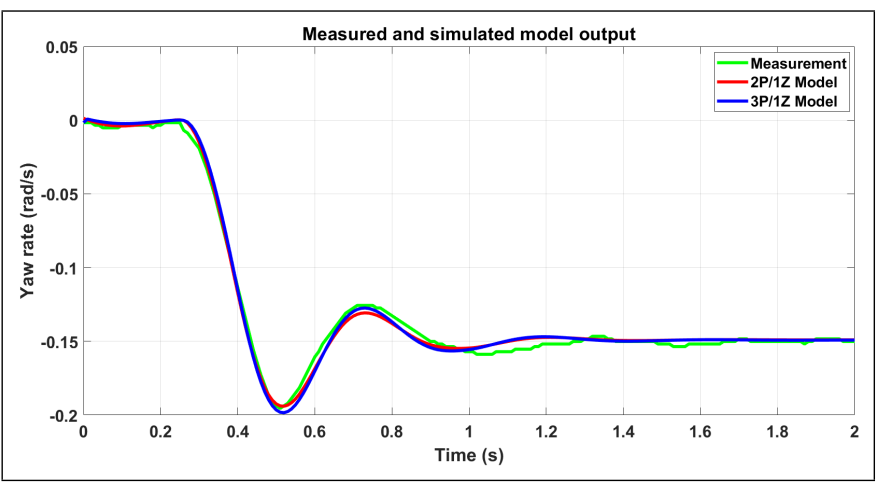

Fig. 2. Comparison of the measured response and the estimated ones.

use different transfer function shapes to approach the vehicle response. After several experiments we can conclude that two poles and one zero suffice to represent the vehicle dynamics influenced by the rear steering. Fig. 2 shows that there is no need to add for example a third pole. Note that the zero characterize the input. The real dynamics of the vehicle are therefore the two identified poles. The problem is that for the same speed value, we may obtain slightly different models. Consequently, in this paper we develop a nominal model based on the experiments carried out for the same speed value, we add a dynamic uncertainty that covers all the model identified, and we isolate the varying parts depending on the speed using our knowledge on vehicle dynamics. The nominal model has the following shape:

$$
G_{n o m}(s)=K_{4 W S} \frac{1+Z_{4 W S}(V) s}{1+2 \frac{\zeta(V)}{\omega_{c}(V)}+\left(\frac{s}{\omega_{c}(V)}\right)^{2}}
$$

Where:

- $G_{\text {nom }}$

- $s$

- $V$

- $Z_{4 W S}(V)=b V$

: the chosen nominal plant,

: Laplace operator,

$:$ the vehicle's speed,

4WS system which depends on $V$, with $b$ being a parameter characterizing the mass and cornering stiffness of the front axle [16],

- $K_{4 W S}(V)=-\frac{V}{L+a V^{2}} \quad$ : the gain generated by the 4WS system which depends on $V$, with $a$ being a parameter characterizing the mass and cornering stiffness of the front and rear axles [16],

- $\zeta(V)=\frac{\zeta_{0}}{\sqrt{1+\left(\frac{V}{V_{c h}}\right)^{2}}} \quad:$ the damping of the vehicle, which depends on $V$, with $\zeta_{0}$ is the natural damping and $V_{c h}$ is the characteristic speed of the vehicle [16],

- $\omega_{c}(V)=\omega_{0} \sqrt{1+\left(\frac{V}{V_{c h}}\right)^{2}}:$ the crossover frequency of the vehicle, which depends on $V$, with $\omega_{0}$ being the natural one [16].

We add a dynamic uncertainty so the set of identified models for the same speed fit into the set of uncertain models. We verify also the validity of this set for other speed values by changing it in the nominal model.

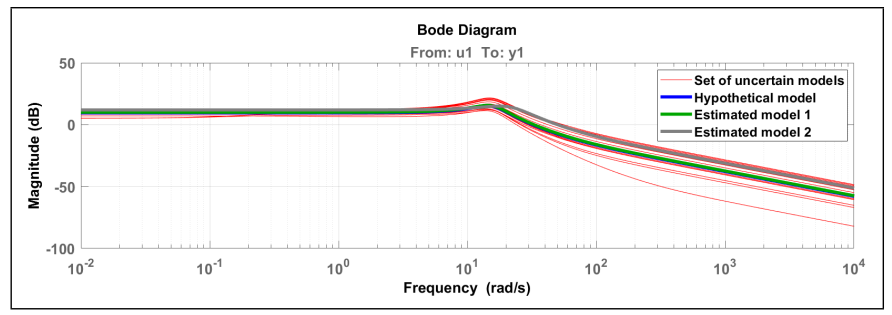

Fig. 3. Set of uncertain models to include the varying nominal model and identified experimental models.

Fig. 3 shows that with a proper dynamic frequency dependent uncertainty applied to the hypothetical speed-dependent 
nominal model, we can include the estimated models from experiments into a set of uncertain models. Fig. 4 shows that

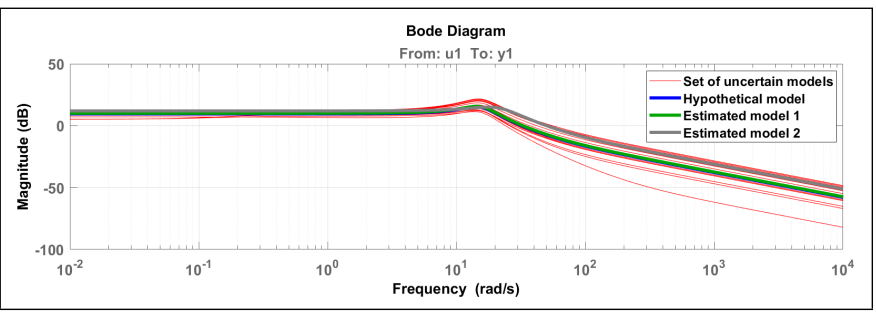

Fig. 4. Set of uncertain models for a speed of $70 \mathrm{~km} / \mathrm{h}$.

this approach remains valid even if we vary the speed value.

The same procedure is carried out for the 4WS actuator only, by considering this time the input/output data at the actuator level only (see Fig. 1). This time a small delay is needed to fit the real dynamics of the actuator as Fig. 5 shows. The overall system is composed then of two poles

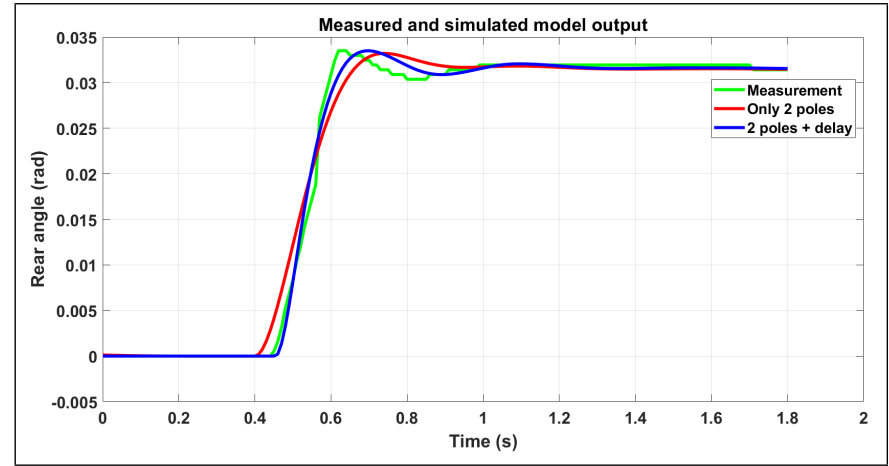

Fig. 5. Comparison of the measured response and the estimated ones for the 4WS actuator.

characterizing the vehicle dynamics, one zero, one delay and two poles characterizing the $4 \mathrm{WS}$ system dynamics.

2) The VDC system identification: We follow the same procedure for the VDC also. However, as we have mentioned, we do not have access to the effective torques applied to the wheels. Here, we apply a torque of $400 N . m$ at each left wheel, and we measure the yaw rate response of the vehicle. Fig. 6 shows that three poles, one zero, and one delay are

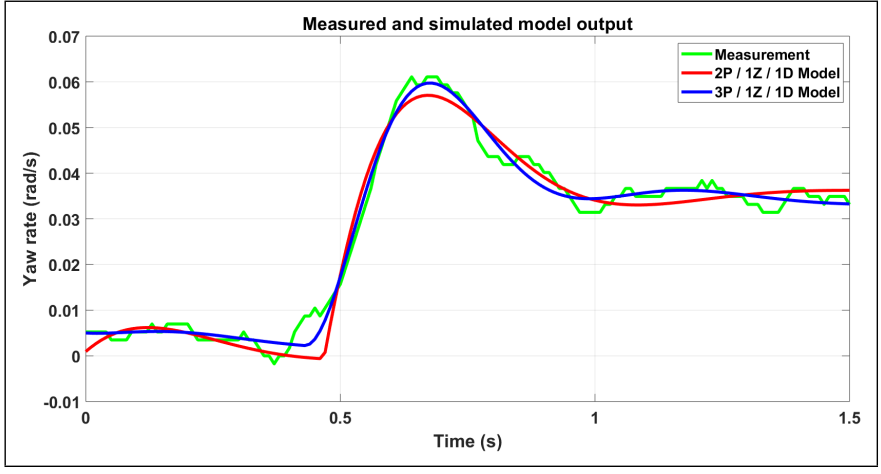

Fig. 6. Comparison of the measured response and the estimated ones for the VDC. needed to represent both the vehicle and the VDC system.

By analyzing the poles and zeros of the influence of the 4WS and the VDC system in Fig. 7, we can see that the complex poles of both dynamic models are close enough.

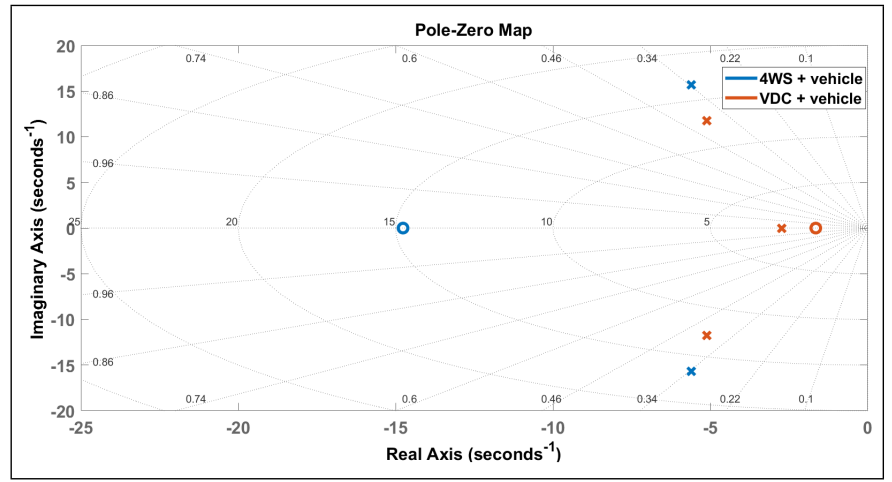

Fig. 7. Poles-zeros analysis.

We can conclude that the complex poles characterize the vehicle dynamics. The remaining pole, zero and pure delay characterize the VDC system. Following again the same procedure, we can develop a hypothetical nominal model augmented by a dynamic uncertainty to cover all the experiments. A high-level controller can be synthesized relying on the uncertain complex poles of the vehicle, and two lowlevel controllers can be synthesized based on the uncertain dynamics of each embedded system and its influence (zeros) on the vehicle dynamics.

\section{B. Analytic Modeling}

To explain the need of an analytic model as well, we should define the control allocation problem. This latter can be defined as follows [17]: find $\vec{u} \in \mathbb{R}^{n}$ such that

$$
\mathbf{B} \vec{u}=\vec{v}
$$

subject to

$$
\left\{\begin{aligned}
& \vec{u}_{\min } \leq \vec{u} \leq \vec{u}_{\max } \\
& \dot{\vec{u}} \leq \dot{\vec{u}}_{\max }
\end{aligned}\right.
$$

where $\mathbf{B} \in \mathbb{R}^{m \times n}$ is a control effectiveness matrix, $\vec{u}_{\text {min }} \in$ $\mathbb{R}^{n}$ and $\vec{u}_{\max } \in \mathbb{R}^{n}$ are the lower and upper position limits, respectively, $\dot{\vec{u}} \in \mathbb{R}^{n}$ is the control rate, $\dot{\vec{u}}_{\max } \in \mathbb{R}^{n}$ is the maximum control rate, $\vec{v} \in \mathbb{R}^{m}$ are the desired accelerations, $n$ is the number of control effectors, and $m$ is the number of axes to control with $n>m$.

Using an optimization procedure, we can see that the distribution will depend closely on the coefficients contained in B. This matrix expresses the influence of each control component on the overall system. It should contain therefore coefficients of the same unit for a right comparison. In our case, we should compare the influence of a steering angle and a braking torque. One could think of transforming the steering angle to an equivalent torque, or the torques to an equivalent steering angle. However, both systems generate first tire forces then a yaw moment. In addition, lateral tire forces and longitudinal ones are coupled and penalize 
each other according to the principle of the friction ellipse [18]. Consequently, for a proper coordination, it is the tire forces that should be distributed optimally by taking into account dynamic saturation due to the friction ellipse [19]. The problem is that we do not have access to tire forces in real time. The control allocation layer outputs will be then transformed to percentages to be applied to the control logic. The distribution algorithm is then based on an analytic vehicle model showing the influence of tire forces.

As we want to control the four brakes, a 4-wheeled vehicle model is needed. As only longitudinal and lateral forces are controllable, vertical motions were ignored making the model planar. However, vertical forces variations have significant influence on tire behavior [18]. These should be taken into account in the tire model [19]. Using Newton's second law of motion, we can find the following equations:

$$
\left\{\begin{aligned}
\frac{d}{d t} \dot{\psi}= & \frac{M_{z_{t o t}}}{J_{z}} \\
M_{z_{t o t}}= & \left(F_{x_{f l}}+F_{x_{f r}}\right) l_{f} \sin \left(\delta_{f}\right) \\
& +\left(F_{x_{f r}}-F_{x_{f l}}\right) \frac{E}{2} \cos \left(\delta_{f}\right) \\
& +\left(F_{x_{r r}}-F_{x_{r l}}\right) \frac{E}{2}-F_{y_{r}} l_{r}
\end{aligned}\right.
$$

Where:

- $J_{z} \quad$ : vehicle's yaw moment of inertia,

- $l_{f / r}:$ distance between the front/rear axle and the vehicle's center of gravity $(\mathrm{CoG})$,

- $E \quad$ : vehicle's track.

\section{ROBUST CONTROL DESIGN}

After several experiments, slightly different models have been obtained. As we have shown in Section II, a dynamic uncertainty has been added to the nominal model to cover all experiments. The reason behind this methodology is to develop a robust controller able to stabilize all chosen uncertain models. In this paper, we compare two well-known control design techniques: $\mathcal{H}_{\infty}$ synthesis and $\mu$ synthesis.

\section{A. $\mathcal{H}_{\infty}$ Synthesis}

$\mathcal{H}_{\infty}$ synthesis is an optimization method to minimize the $\mathcal{H}_{\infty}$ norm of the augmented plant containing weight functions. In this paper, three weight functions are considered: $\mathbf{W}_{1}, \mathbf{W}_{\mathbf{2}}$ and $\mathbf{W}_{\mathbf{3}}$ penalizing the error signal, control signal and output signal respectively as Fig. 8 shows. The standard

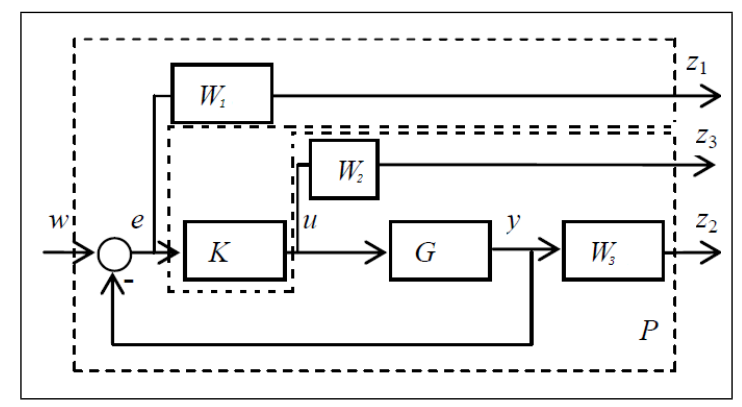

Fig. 8. Augmented plant for $\mathcal{H}_{\infty}$ synthesis (adapted from [20]).
$\mathcal{H}_{\infty}$ problem is then defined as: "Considering a positive real parameter $\gamma>0$, find a controller $K$ that satisfies $P \star K$ is asymptotically stable, and $\|P \star K\|_{\infty}<\gamma$, if it exists." With * is "The Product of Redheffer". A stabilizing controller $K(s)$ can be synthesized by solving algebraic Riccati equations minimizing the cost function $\gamma$ [21].

\section{B. $\mu$ Synthesis}

The uncertainty block may be structured [22]. This led Doyle to propose in [23] the concept of "structured singular value" commonly referred to " $\mu$ ". The structured singular value $\mu_{\Delta}(T)$ of a complex-valued matrix $T$ with respect to the perturbation structure in $\Delta$ in defined as [22]:

$$
\mu_{\Delta}(T):=\frac{1}{\min \{\bar{\sigma}(\Delta): \Delta \in \mathcal{D}, \operatorname{det}(I-T \Delta)=0\}}
$$

With $\bar{\sigma}$ is the largest singular value, and $\mathcal{D}$ is the class of constant complex-valued matrices having null off-diagonal coefficients. Consequently, the structured singular value $\mu_{\Delta}(T)$ is the inverse of the largest singular value of the smallest perturbation $\Delta \in \mathcal{D}$ that makes $(I-T \Delta)$ singular. Thus, the larger is $\mu_{\Delta}(T)$, the smaller is the perturbation $\Delta$ which is needed to make $(I-T \Delta)$ singular.

Unfortunately, there is no analytic method to calculate a $\mu$-optimal controller due to computational difficulties [20],[22]. Nevertheless, an approximate solution, known as "DK-iteration", is generally used using Matlab ${ }^{\circledR}$ [24]. This approach relies on the property:

$$
\mu_{\Delta}(T) \leq \bar{\sigma}\left(D T \bar{D}^{-1}\right)
$$

With $D$ is a scaling matrix chosen so it commutes with $\Delta$ : $D \Delta=\Delta D$. The iterative process goes as follows [25]:

1) Synthesize an $\mathcal{H}_{\infty}$ controller for the scaled problem with a fixed $D$ :

$$
\min _{K}\left(\left\|D T D^{-1}\right\|_{\infty}\right)
$$

2) Find $D(j \omega)$ to minimize at each frequency $\bar{\sigma}\left(D(j \omega) T D^{-1}(j \omega)\right)$

3) Fit the magnitude of each element of $D(j \omega)$ to a stable and minimum-phase transfer function. Go to step 1 .

The iteration process should be stopped if $\left\|D N D^{-1}\right\|_{\infty} \leq 1$ or if the norm no longer decreases.

\section{Comparison}

Here, we keep the same weight functions and the same uncertainty modeling. We apply the $\mathcal{H}_{\infty}$ control design and then the $\mu$ control design to the synthesis of the highlevel controller based on the uncertain poles of the vehicle dynamics. A step response of closed loops of both controllers applied to the set of identified models form experiments is illustrated in Fig. 9. It is clear that $\mu$ synthesis is more robust than the $\mathcal{H}_{\infty}$ synthesis as expected. However, the $D K$-iteration takes a greater amount of calculation time to synthesize the controller, and leads to much higher order than the $\mathcal{H}_{\infty}$ provides [26]. Nevertheless, safety is our major goal in vehicle motion control. We rather prefer to be cautious and 


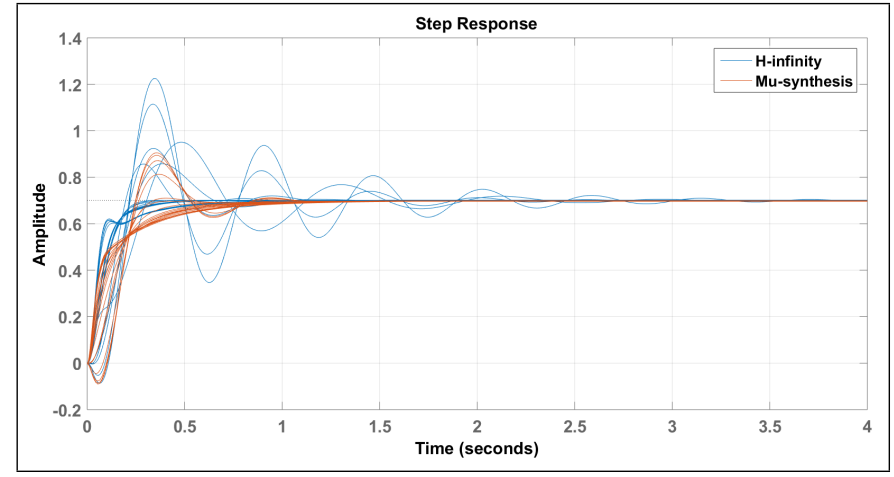

Fig. 9. Comparison of $\mathcal{H}_{\infty}$ synthesis and $\mu$ synthesis.

opt for the most robust controller. The $\mu$-synthesis is therefore selected for the high-level controller, and both low-level controllers (4WS and VDC). Each controller is carefully reduced afterwards to avoid implementation issues. We redo the same procedure for different speed values. Controllers' parameters are then extrapolated. A gain-scheduling $\mu$-robust controllers are applied in what follows.

\section{CONTROL ALLOCATION}

As we have detailed in equation (2), the problem is how to distribute the desired global control effort calculated by the high-level controller into the effectors. In our case, the control vector contains the controllable tire forces:

$$
\vec{u}=\left[\begin{array}{c}
F_{x_{f l}} \\
F_{x_{f r}} \\
F_{x_{r l}} \\
F_{x_{r r}} \\
F_{y_{r}}
\end{array}\right]
$$

$\vec{u}_{\min }$ and $\vec{u}_{\max }$ reflect tire limits with respect to the friction ellipse concept [19]:

$$
\left\{\begin{array}{l}
F_{x} \leq \sqrt{\left(\mu F_{z}\right)^{2}-F_{y}^{2}} \\
F_{y} \leq \sqrt{\left(\mu F_{z}\right)^{2}-F_{x}^{2}}
\end{array}\right.
$$

We suppose that the friction coefficient and the vertical loads can be estimated using for example the methods detailed in [27]. The desired acceleration $\vec{v}$ contains in this case the global yaw moment $M_{z_{t o t}}$. To complete the problem definition described in (2), $\mathbf{B}$ is filled by geometric relations between the vehicle and its tires using equation (5):

$$
\mathbf{B}=\left[\begin{array}{lllll}
b_{1,1} & b_{1,2} & -\frac{E}{2} & \frac{E}{2} & -l_{r}
\end{array}\right]
$$

where:

$$
\begin{aligned}
& \text { - } b_{1,1}=l_{f} \sin \left(\delta_{f}\right)-\frac{E}{2} \cos \left(\delta_{f}\right), \\
& \text { - } b_{1,2}=l_{f} \sin \left(\delta_{f}\right)+\frac{E}{2} \cos \left(\delta_{f}\right) .
\end{aligned}
$$

Due to interfaces issues, the control allocation layer is not located between the high-level controller and the low-level controllers as in [12] for example. Here, the control allocation algorithm acts more like a supervisor that quantifies the amount of effectiveness that each subsystem should satisfy. Therefore, a simple integrator has been added upstream the control allocation algorithm to transform the yaw rate error into the global yaw moment required to steer the vehicle. Downstream the control allocation, the fractions $\frac{\left|b_{i, j} F_{x_{i, j}}\right|}{\left|M_{z_{\text {tot }}}\right|}$ and $\frac{\left|b_{1,5} F_{y_{r}}\right|}{\left|M_{z_{\text {tot }}}\right|}$ are determined to evaluate the percentage that each actuator should satisfy to control to overall vehicle. Only these percentages are directly implemented between the high-level controller and the low-level controllers.

Regarding the online optimization solver, various techniques have been compared in [9]. It has been shown that the Weighted Least Squares (WLS) formulation based on Active Set Algorithms (ASA) solves the problem rapidly with good precision and reach the optimum in a small finite number of iterations. The WLS solves the global problem in a one stage ASA by means of different weights to determine the importance of each objective [28]. This gives the following expression:

$$
\begin{aligned}
\vec{u}_{\text {opt }}=\arg \min _{\vec{u}_{\min } \leq \vec{u} \leq \vec{u}_{\max }} & \left\|\mathbf{W}_{\mathbf{u}}\left(\vec{u}-\vec{u}_{p}\right)\right\|^{2} \\
& +\gamma\left\|\mathbf{W}_{\mathbf{v}}(\mathbf{B} \vec{u}-\vec{v})\right\|^{2}
\end{aligned}
$$

Where:

- $\vec{u}_{p} \quad$ : preferred control vector,

- $\mathbf{W}_{\mathbf{u}}$ : non-singular weighting matrix affecting control distribution among the actuators,

- $\mathbf{W}_{\mathbf{v}}$ : non-singular weighting matrix affecting the prioritization among the virtual control components when $\mathbf{B} \vec{u}=\vec{v}$ cannot be attained due to actuator constraints.

\section{RESULTS}

Two major features should be verified in this section: control robustness and control allocation benefits. Regarding control robustness, we compare controllers developed and validated by co-simulation using a high-fidelity software as in [9],[12],[27], and the controllers developed in this paper using an experimental maneuver. However, to show the benefits of control allocation algorithms, we only use simulation of the validated identified models by experiment. We did not carry experimental maneuvers due to lack of time and unavailability of prototypes for a long period of time.

\section{A. Control Robustness}

In previous researches [9],[12],[27], the problem defined here has been solved and validated using co-simulation of Matlab/Simulink ${ }^{\circledR}$ and LMS Imagine.Lab AMESim ${ }^{\circledR}$. A high-fidelity vehicle model with 15 degrees of freedom provided by AMESim has been adapted to meet the nominal parameters of the real vehicle prototype. This has led the authors to synthesize a relatively simpler and structured $\mathcal{H}_{\infty}$ controller as a high-level controller, and adaptive gains as low-level controllers based on a linear tire model with varying-parameters [19].

We carried an experiment of a slalom at a speed of $50 \mathrm{~km} / \mathrm{h}$. By applying the experimental input signals into the co-simulation platform, we obtain the Fig. 10. 


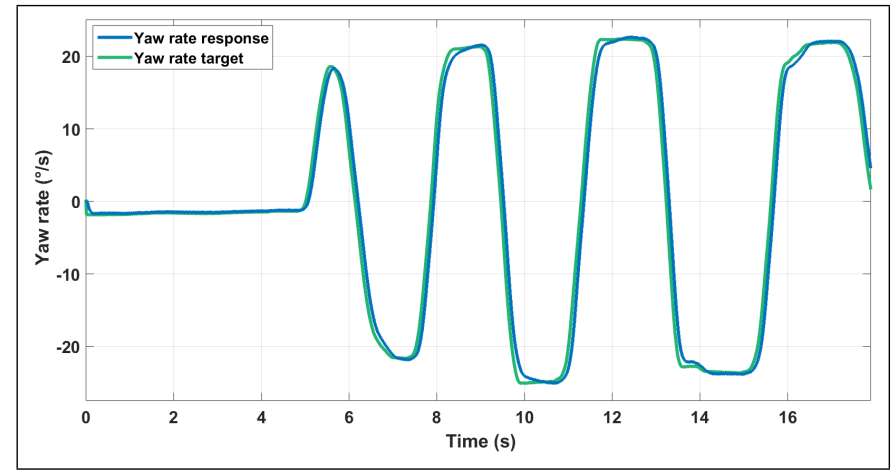

Fig. 10. Co-simulation of the yaw rate control.

This suggests good performance of this simpler logic and lead us to validate it. Now when we apply the same control logic to the identified models, we obtain the Fig. 11.

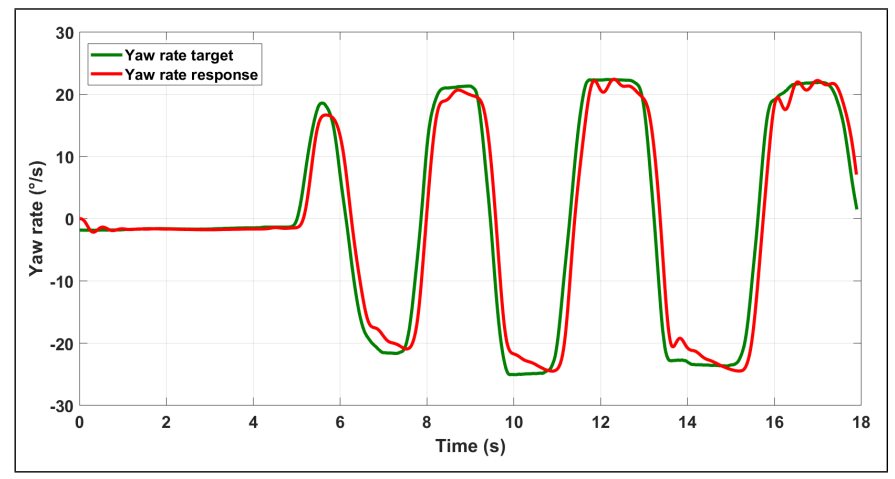

Fig. 11. Yaw rate control using the identified model.

The control starts loosing its precision even in this simple maneuver. Note also the fluctuations that start showing up which is an indication of the limit of stability. One could suggest that the identified models may be faulty or too restrictive. That is why we carried out the slalom maneuver as our experiment by using the robust controllers developed in this paper. The comparison between the experimental response and the simulated one is illustrated in Fig.12:

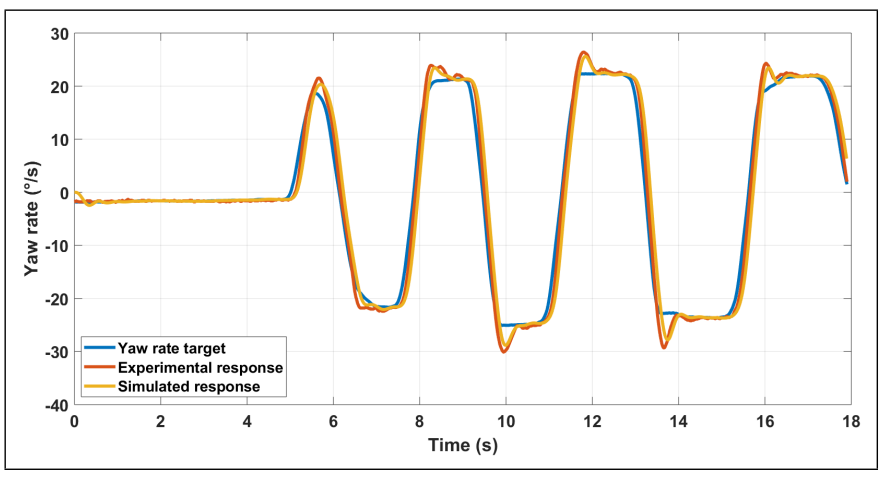

Fig. 12. Comparison of the experimental response and the simulated one.

The simulated response is almost identical to the experimental one. In addition, the control is more precise and stable. The overshoots are almost inevitable in order to obtain good performances. This proves that the identified models are more representative than the high-fidelity software, and therefore the need to develop new identified models for advanced chassis systems, especially in an MBD framework. These models can vary from an experiment to another. To overcome this, dynamic uncertainties can be applied to a chosen nominal model, and a robust controller can be developed based on the set of uncertain models identified to ensure successful real-life control applications.

\section{B. Benefits of Optimal Control Allocation}

In the over-actuation context, several discussions have led to the need of an upstream coordination by means of optimization-based control allocation algorithms [5]. Unfortunately, due to lack of time, severe situations as faulttolerance or actuators' saturation were not experimented. Nevertheless, as the identified models have proven their precision with respect to the real behavior of the overactuated vehicle, simulations can be conducted to foresee the performance of the control allocation layer.

The industrial common practice though is to activate one system at a time in specific ranges in order to avoid subsystems interactions [5]. This limits the overall potential of the car. In order to show the benefits of control allocation, we should test the over-actuated vehicle in its limits of handling, but in a realistic scenario. Let us suppose for example a sporty vehicle going at a speed of $70 \mathrm{~km} / \mathrm{h}$. The vehicle encounter a progressive cornering where it should steer. The yaw rate target resembles then to a ramp. We first test the vehicle equipped by only the 4WS system. Then, we add the VDC with a simple downstream coordination strategy consisting in deactivating the 4WS system when the vehicle loses control and activating the VDC as an emergency backup. Finally, we add the control allocation layer to optimally coordinate both systems when they are activated at the same time. Fig. 13 shows the results.

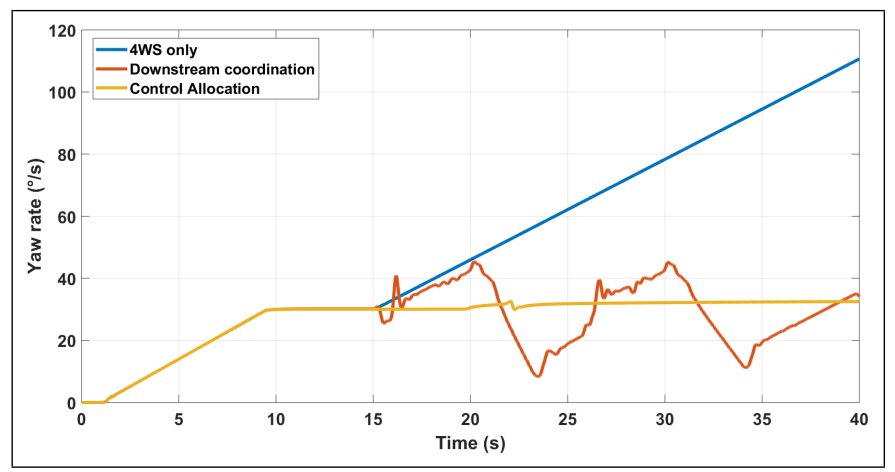

Fig. 13. Comparison of systems coordination methods.

Note that in this situation we let the front steering angle continue growing to see if the chassis systems can detect the limit of handling and saturate the command. The 4WS system saturates around $t=15 \mathrm{~s}$. If only the $4 \mathrm{WS}$ system is activated, the front steering angle influence takes over which make the vehicle exceed the limits of handling. Regarding the simple downstream coordination, switching controllers 
in this severe maneuver can reduce the amount of yaw rate but destabilizes the vehicle as soon as the 4WS system is saturated. Thanks to the control allocation layer, these problems can be overcome. Both subsystems are activated at the same time. Optimal coordination is ensured not only when actuators are saturated, but also when the friction ellipse limits are not respected.

\section{CONCLUSIONS}

In this paper, two major aspects have been discussed regarding passenger cars motion control: robustness of the control especially when the subsystems provided by the suppliers come in a black-box, and the coordination strategy once the vehicle becomes over-actuated. Control synthesis have been carried using $\mu$-robustness framework, and control allocation algorithms have been applied to optimally coordinate the embedded systems.

Contrary to previous researches, controllers have been synthesized using identified models from experiments. Indeed, today's high-fidelity software fail to depict the real dynamics of advanced chassis systems. This proves the limits of co-simulation procedure for future vehicles and the need to upgrade high-fidelity software or adopt the design methodology presented in this paper. Another solution could be tackling the "openness" problem by both manufacturers and suppliers to design open-boxes modules for an efficient overall vehicle motion control logic without jeopardizing the intellectual properties of each stakeholder.

Control allocation algorithms also proves their benefits when it comes to expanding the potential of the vehicle. As we are advancing towards the full-autonomous driving, more systems are intended to be implemented. The more numerous the embedded systems within the same car will get, the more relevant these algorithms would become as the most important interface between the manufacturer and its suppliers. These algorithms need to be tested by experiments also to evaluate their real performances and limits. This represent our actual ongoing work with Renault. We expect better coordination of chassis systems by the end of the experiments, and the possibility of coming up with standardization for over-actuated vehicles.

\section{REFERENCES}

[1] M. Gerard and E.Y. Lopes, Global Chassis Control and Braking Control using Tyre Forces Measurement, TU Delft, 2011.

[2] W. Shi, Mohamed Baker Alawieh, Xin Li, Huafeng Yu, Algorithm and hardware implementation for visual perception system in autonomous vehicle: A survey, Integration, vol. 59, 2017, pp. 148-156.

[3] W. Shi, M. B. Alawieh, X. Li, H. Yu, N. Arechiga and N. Tomatsu, Efficient Statistical Validation of Machine Learning Systems for Autonomous Driving, Proceedings of the 35th International Conference on Computer-Aided Design (ICCAD 16), ACM, 2016, Austin, Texas, pp. 36:1-36:8.

[4] A. Singh, A. Kumar, R. Chaudhary and R. C. Singh, Study of 4 Wheel Steering Systems to Reduce Turning Radius and Increase Stability, International Conference of Advance Research and Innovations (ICARI2014), 2014.

[5] M. Kissai, B. Monsuez and A. Tapus, Review of integrated vehicle dynamics control architectures, 2017 European Conference on Mobile Robots (ECMR), Paris, 2017, pp. 1-8.
[6] M. Velardocchia and A. Vigliani, Control systems integration for enhanced vehicle dynamics, The Open Mechanical Engineering Journal, vol. 7, 2013, pp. 58-69.

[7] J.-X. Wang, N. Chen, D.-W. Pi and G.-D. Yin, Agent-based coordination framework for integrated vehicle chassis control, Proceedings of the Institution of Mechanical Engineers, Part D: Journal of Automobile Engineering, vol. 223, no. 5, pp. 601-621, 2009.

[8] Marc Bodson, Evaluation of Optimization Methods for Control Allocation, Journal of Guidance, Control, and Dynamics, Vol. 25, No. 4 (2002), pp. 703-711.

[9] M. Kissai, X. Mouton, B. Monsuez, D. Martinez and A. Tapus, Complementary Chassis Systems for Ground Vehicles Safety, 2018 IEEE Conference on Control Technology and Applications (CCTA), Copenhagen, 2018, pp. 179-186.

[10] H. Jing, F. Jia, H. Liu and J. Sun, Multi-objective optimal control allocation for a four-wheel-independent-drive electric vehicle, 2017 36th Chinese Control Conference (CCC), Dalian, 2017, pp. 9543-9547.

[11] B. Shyrokau, D. Wang, Control allocation with dynamic weight scheduling for two-task integrated vehicle control, Proc. of the 11th International Symposium on Advanced Vehicle Control, Seoul, Korea, 2012.

[12] M. Kissai, X. Mouton, B. Monsuez, D. Martinez and A. Tapus, Optimizing Vehicle Motion Control for Generating Multiple Sensations, 2018 IEEE Intelligent Vehicles Symposium (IV), Changshu, 2018, pp. 928-935.

[13] G. Nicolescu, P. J. Mosterman, Model-Based Design for Embedded Systems, CRC Press, 2010.

[14] S. Brennan and A. Alleyne, Integrated vehicle control via coordinated steering and wheel torque inputs, Proceedings of the 2001 American Control Conference. (Cat. No.01CH37148), Arlington, VA, USA, 2001, pp. 7-12 vol.1.

[15] E. Ono, K. Takanami, N. Iwama, Y. Hayashi, Y. Hirano, Y. Satoh, Vehicle integrated control for steering and traction systems by $\mu$ synthesis, Automatica, vol. 30, issue 11, 1994, pp. 1639-1647.

[16] A. Soltani, Low Cost Integration of Electric Power-Assisted Steering (EPAS) with Enhanced Stability Program (ESP), PhD thesis, Cranfield University, 2014.

[17] T. A. Johansen and T. I. Fossen, Control Allocation - A survey, in Automatica, Vol. 49, Issue 5, May 2013, pp. 1087?1103.

[18] H.B. Pacejka, Tyre and Vehicle Dynamics, Second Edition. Elsevier, Butterworth-Heinemann, 2006.

[19] M. Kissai, B. Monsuez, A. Tapus and D. Martinez, A new linear tire model with varying parameters, 2017 2nd IEEE International Conference on Intelligent Transportation Engineering (ICITE), Singapore, 2017, pp. 108-115.

[20] A. Vasičkanmová, M. Bakošová, E. Čirka, M. Kalúz, Comparison of Robust Control Techniques for Use in Continuous Stirred Tank Reactor Control, IFAC-PapersOnLine, vol. 48, issue 14, 2015, pp. 284-289.

[21] J. C. Doyle, K. Glover, P. P. Khargonekar and B. A. Francis, Statespace solutions to standard $\mathcal{H}_{2}$ and $\mathcal{H}_{\infty}$ control problems, in IEEE Transactions on Automatic Control, vol. 34, no. 8, pp. 831-847, Aug. 1989.

[22] Wal, van de, M. M. J., Actuator control with H-infinity and musynthesis. (DCT rapporten; Vol. 1995.062). Eindhoven: Technische Universiteit Eindhoven, 1995.

[23] J. Doyle, Analysis of feedback systems with structured uncertainties, in IEE Proceedings D - Control Theory and Applications, vol. 129, no. 6, pp. 242-250, November 1982.

[24] G. J. Balas, J. C. Doyle, K. Glover, A. Packard, R. Smith, $\mu$-analysis and synthesis toolbox: for use with Matlab ${ }^{\circledR}$, user's guide version 3. The MathWorks Inc., Natick, MA, USA, 1998.

[25] S. Skogestad, I. Postlethwaite, Multivariable Feedback Control: Analysis and Design, 2nd Edition, John Wiley \& Sons, Inc., 2005.

[26] F.L. Santos and A.A. Filho, Comparison of Robust Control Techniques for Use in Active Suspension Systems, ABCM Symposium Series in Mechatronics, vol. 5, pp. 270-279, 2012.

[27] M. Kissai, B. Monsuez, X. Mouton, D. Martinez and A. Tapus, Importance of Vertical Dynamics for Accurate Modelling, Friction Estimation and Vehicle Motion Control, 2018 21st International Conference on Intelligent Transportation Systems (ITSC), Maui, HI, USA, 2018, pp. 1370-1377.

[28] O. Harkegard, Efficient active set algorithms for solving constrained least squares problems in aircraft control allocation, Proceedings of the 41st IEEE Conference on Decision and Control, Vol. 2, 2002, pp. $1295-1300$. 